\title{
Microencapsulation of red palm oil as an oil-in-water emulsion with supercritical carbon dioxide solution-enhanced dispersion
}

\begin{abstract}
Feasibility of microencapsulating red palm oil (RPO) with solution enhanced-dispersion by supercritical carbon dioxide (SEDS) without using high-temperatures or organic solvents was assessed. RPO prepared as oil-in-water $(\mathrm{o} / \mathrm{w})$ emulsion $(11.7 \%$ RPO, $69.9 \%$ water, 3.5\% sodium caseinate, $14.0 \%$ maltodextrin, $1.0 \%$ soy lecithin) could be encapsulated at all conditions (100-150 bar, $40-60{ }^{\circ} \mathrm{C}$, feed injection flow rate $2.5 \mathrm{~mL} / \mathrm{min}$ ). Microcapsules produced with the SEDS method $\left(125 \mathrm{bar}, 50{ }^{\circ} \mathrm{C}, \mathrm{CO}_{2}\right.$ feed $\left.150 \mathrm{~L} / \mathrm{h}\right)$ were flowable, spherical powders $(d=5.8 \mu \mathrm{m}, \sigma=2.8 \mu \mathrm{m})$ containing $31.6 \%$ oil with $92.1 \% \mathrm{ME}, 82.7 \% \mathrm{RE}$ for carotenes and $94.3 \% \mathrm{RE}$ for vitamin $\mathrm{E}$, whereas those from spray drying were irregularshaped particles $(d=16.6 \mu \mathrm{m}, \sigma=8.6 \mu \mathrm{m})$ containing $39 \%$ oil, $79 \% \mathrm{ME}$, and having similar $\mathrm{RE}$ values. The SEDS method allows microencapsulation of food oils prepared as $\mathrm{o} / \mathrm{w}$ emulsions without thermal stress or organic solvents.
\end{abstract}

Keyword: Red palm oil; scCO2; SEDS; Particle formation; Microcapsule; Spray drying 